\title{
CLINICAL OUTCOMES OF LOW LEVEL LASER THERAPY (LLLT) IN THE MANAGEMENT OF TEMPOROMANDIBULAR JOINT DISORDERS
}

\author{
Mohammad Abdelhamied Shuman*
}

\begin{abstract}
Temporomandibular disorder (TMD) is a collective term for a number of clinical signs and symptoms involving the masticatory muscles, the temporomandibular joint (TMJ), and associated structures. ${ }^{1}$ pain relief and reestablishment of normal jaw function are the main goals of conservative (noninvasive or minimally invasive) management of (TMD). These have ranged from occlusal adjustment, splint therapy, arthrocentesis, arthroscopy, laser therapy, acupuncture, cryotherapy, and physical exercises. Low-level laser therapy (LLLT) has been used for pain control and healing. Use of LLLT for TMD has been controversial, and shortcomings have been found in previous studies. The aim of this study was to evaluate the efficacy of (LLLT) in the treatment of (TMD) in relation to pain intensity and jaw movements. In this randomized, double-blind clinical trial, 40 patients with TMDs were randomly divided into laser and placebo groups 20 patients in each. In the laser group, twenty patients received 6 sessions of LLLT (2 times a week for 3 weeks) with semi conductive diode laser (gallium arsenide; $904 \mathrm{~nm}, 0.6 \mathrm{~W}, 60 \mathrm{~s}, 4 \mathrm{~J} / \mathrm{cm} 2$ ). The same procedure was done with the 20 patients of the control group but by using inactive beaming light. Pain intensity, and range of motion were assessed before and immediately after 1, 2 and 3 weeks, 1, 3 and 6 months. Statistically significant results were achieved in all study parameters. It was concluded that LLLT promoted satisfactory results in reducing the pain intensity, and improvement in the range of jaw motion. Hence it is an effective and efficient treatment method for TMDs.
\end{abstract}

\section{INTRODUCTION}

Temporomandibular disorder (TMD) is a collective term traditionally used to describe multiple disorders, including intracapsular disorders, true abnormalities of the temporomandibular joint (TMJ), and muscular disorders or myofascial pain dysfunction (MPD) syndrome. TMD is a major cause of non-dental pain in the orofacial region. In the adult non-patient population, approximately $33 \%$ reported at least one TMD symptom, and clinical findings revealed at least one TMD sign in 40\%$75 \%$ of the population ${ }^{(1)}$. It can be a very painful condition, leading to significant deterioration in the patient's quality of life. The primary symptoms associated with TMD include facial muscle pain, preauricular (TMJ) pain, TMJ sounds (jaw clicking, popping, catching, and locking), limited mouth opening, and increased pain associated with chewing. The secondary symptoms are earache, headache, and neck ache ${ }^{(2)}$.
Non-surgical treatment of TMDs continues to be the most effective way of managing over $80 \%$ of patients, which include psychotherapy or behavioral therapy, pharmacotherapy, occlusal splint therapy and various physical therapies like thermal therapy, acupuncture, electrical stimulation, ultrasound therapy, physiotherapy and low intensity laser therapy. ${ }^{(3)}$

LLLT, a term created by Oshiro and Calderhead in 1988, means 'Low Level Laser Therapy' and has been investigated and used clinically for about 30 years. Its basic effects are bio-stimulative, regenerative, analgesic and antiinflammatory. It also seems to act on the immune, circulatory and haematological systems. In addition, LLLT appears to have a virustatic and bacteriostatic effect. ${ }^{(4)}$

The relative clinical efficacy of LLLT for the treatment of TMD is controversial. Some authors reported the efficacy of LLLT to be superior to

\footnotetext{
* Ass. Professor of Oral \& Maxillofacial Surgery, Faculty of Dental Medicine, Al-Azhar University. Assiut. Egypt.
} 
placebo therapy ${ }^{(5-12)}$, while, others found no significant differences between LLLT and placebo for the measures of TMD ${ }^{(13-15)}$. However the greatest advantage of continuing to test laser application for TMD management is its non-invasive, cost effective, does not have any known side effects and less harmful characteristics. The aim of this study was to evaluate the effectiveness and the outcome of LLLT on relieving pain and improving function in TMD patients in a double-blind placebo control trial.

\section{MATERIALS AND METHODS}

Patients were selected for the study among the patients visiting the department of oral and maxillofacial surgery, Al-Azhar University for the treatment of TMD. After obtaining institutional approval (Al-Azhar University Hospital, Egypt). All patients were informed about the procedure in detail and signed informed consent.

Patients with TMD were randomly selected after thorough examination that fulfilled the requirements on the basis of exclusion and inclusion criteria according to the Research Diagnostic Criteria for TMD. ${ }^{(16,17)}$. Panoramic radiographs were taken for all the patient to rule out any gross anatomical deformity in relation to the TMJ.

The criteria for patients included in the study were a chief complaint of acute pain in the joint, Presence of reciprocal joint clicking during jaw opening and closing that limited the mouth opening and not having medical or pharmacological treatment for TMDs.

The exclusion criteria were painless joint sounds, disc displacements without reduction. Patients with TMJ pain due to degenerative systemic diseases (e.g. rheumatoid arthritis, fibromyalgia), those who received analgesic or antidepressant medicine or underwent any other form of treatment for TMD were excluded from the study. Occlusal factors of risk, toothache, neuralgia or local skin infection over the tenderness spot of the masseter and temporal muscles, Patient wearing of full dentures, cases with congenital abnormality, neoplastic conditions were excluded. The presence of major psychological disturbances and restriction for the use of LASER were also exclusion criteria.

A total of 40 patients ( 28 female, 12 male) ranged in age from 18 to 58 years (mean, 42.9 years). The patients were randomly assigned to two groups: Group 1 (20 patients): patients receiving real LLLT (experimental group). Group 2 (20 patients): patients receiving inactive laser (placebo group).

All patients were treated with six sessions twice weekly for 3 consecutive weeks of LLLT. DenLase 980/7 Diode Laser Therapy System (Fig. 1), a Class IV laser, producing semi-conductive (diodic) gallium arsenide (GaAs) laser (input: $5 \mathrm{~V}-14 \mathrm{~A}$, visible output: $1 \mathrm{~m} \mathrm{~W} \max @$ 630-670 nm, invisible output: 7 W max @ 800-990 nm manufactured by (China Daheng Group Inc.) was used in the study. LLLT (wavelength: $904 \mathrm{~nm}$, mean output power: $0.6 \mathrm{~W}$, duration: 60 seconds, dosage: $6 \mathrm{~J} / \mathrm{cm}^{2}$ ) was applied to all the tender points selected during examination. ${ }^{(18,19)}$ The subjects and the clinician used protective eyewear. The therapeutic LLLT application was achieved through direct contact of the probe to the skin. The laser beam was delivered through a handheld suitable laser probe. The probe was placed perpendicular directly on to the skin behind, in front of, and above the joint area (Fig. 2), and into the external acoustic meatus (Fig. 3). It was also applied over the painful muscles like masseter and temporalis. (Fig. $4 \& 5$ ).

Intra-orally the LLLT was applied onto the masseter, anterior border of the mandibular ramus, attachment of temporalis (Fig. 6), posterior and superior to the molars in the buccal vestibule over the lateral pterygoid muscle and on the lingual aspect of the posterior mandible over the medial pterygoid muscle. Each tender point was exposed to LLLT. ${ }^{(20)}$ 


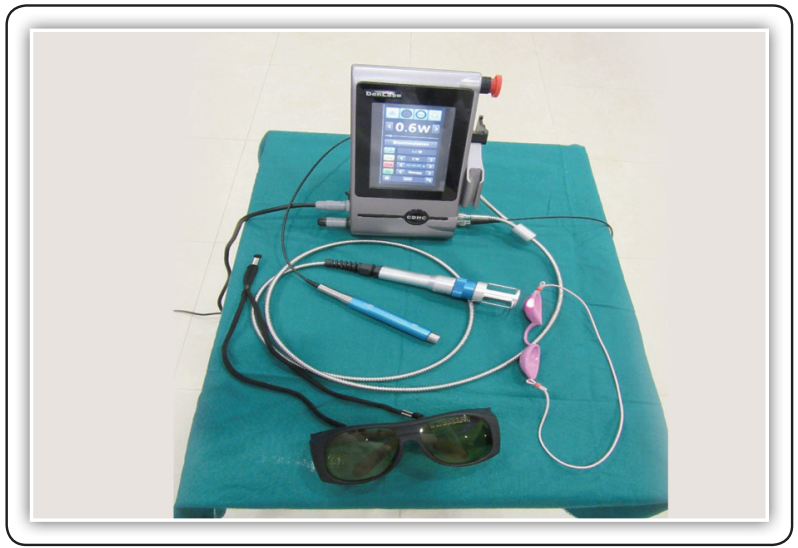

FIG (1) DenLase 980/7 Diode Laser Therapy System

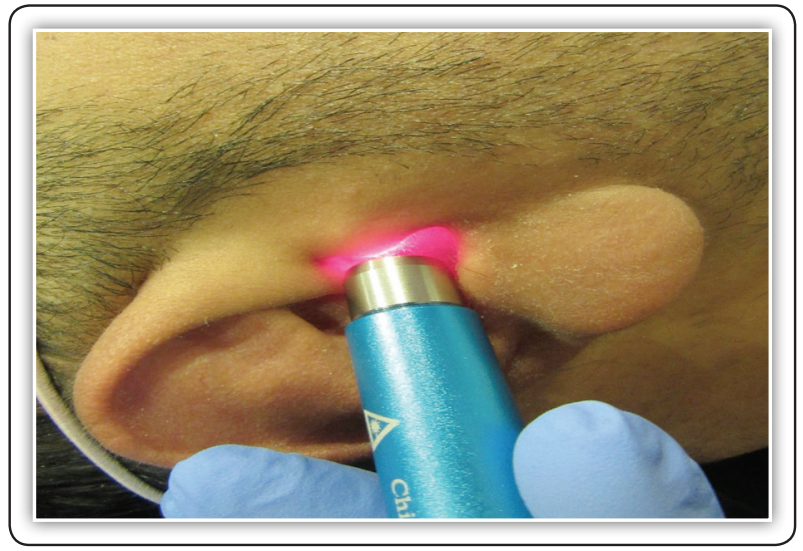

FIG (3) Laser application into the external acoustic meatus

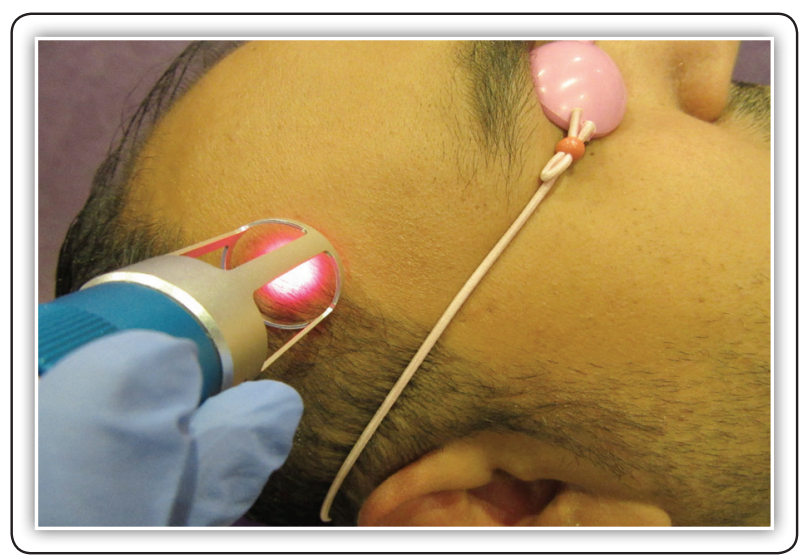

FIG (5) Laser application over temporalis muscle

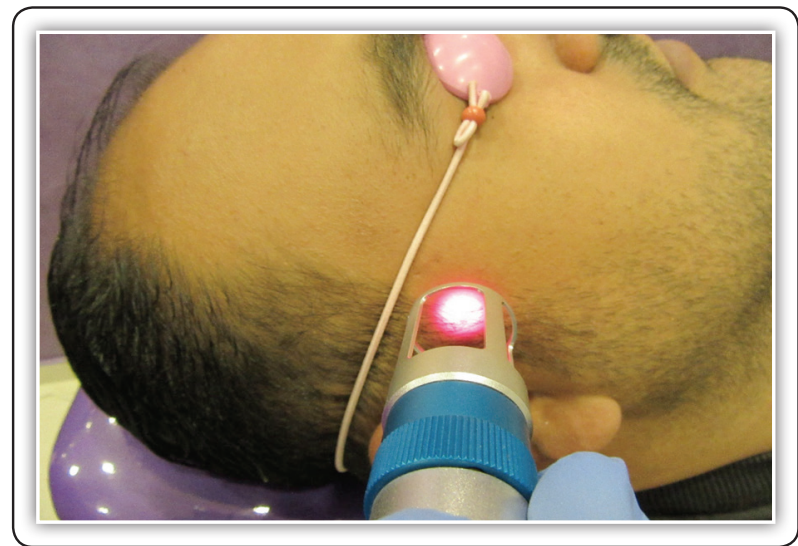

FIG (2) Laser application around the joint area

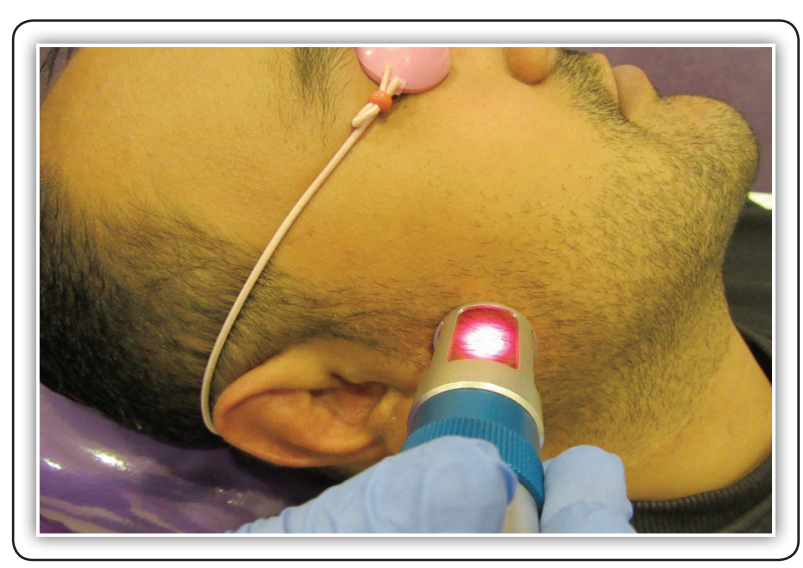

FIG (4) Laser application over masseter muscle

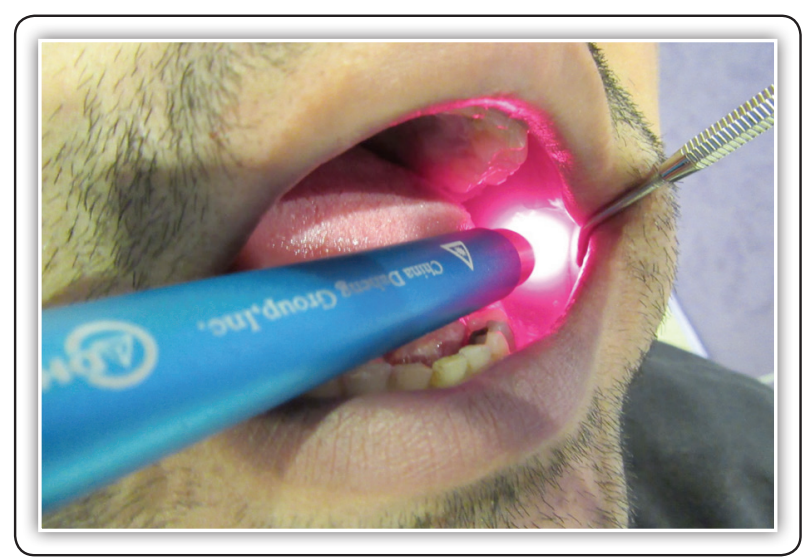

FIG (6) Laser application over intraoral regions of intrest 
Pain intensity was recorded in $\mathrm{mm}$ on a 100 mm Visual Analogue Scale (VAS) which allows the quantification of pain intensity. The VAS is a measurement of pain, in which all individuals scored their pain on a horizontal line measuring $100 \mathrm{~mm}$, where the left end means "no pain" and the right end indicates "the worst pain imaginable".

The patient was asked to open his/her mouth as much as possible for the measurement of maximal active mouth opening. With a digital Vernier caliper, the total mouth opening was recorded, with the measure being performed from the incisal of the upper incisors to the incisal of the lower incisors.

All data were recorded before and immediately after 1, 2 and 3 weeks, 1, 3 and 6 months from the first session.

All the evaluations were performed by an independent investigator who had been trained to do these procedures beforehand. To have a doubleblind study, neither the patient nor the evaluator was aware of the group the participant was assigned to. After completing the study, the subjects in the placebo group who tended to continue treatment received another form of therapy for TMD (occlusal appliance therapy, laser therapy, or pharmacologic therapy). . During the study, patients were instructed not to take systemic medication for TMD.

Microstat7 for windows statistical package (Microstat Co.) was used for statistical analysis in this study. One-Way ANOVA was used to compare between time intervals in each group followed by calculating Least Significant Difference (LSD) for paired comparisons between each interval in the same group. Independent Student " $t$ " test was used to compare both groups in each interval.

Difference was considered statistically significant when $\mathrm{p}<0.05$.

\section{RESULTS}

The scores of the parameters evaluated before and immediately after 1,2 and 3 weeks, 1, 3 and 6 months from the first session (Tab. $1 \& 2$ ).

\section{Pain intensity:}

Pain intensity was evaluated in $\mathrm{mm}$ on a 100 mm Visual Analogue Scale (VAS) which showed no statistically significant difference between pain score values in both groups preoperatively. The pain scores in group 1 was statistically significantly lower than group 2 after 1 week postoperatively until the end of the follow up period. In group 1 (laser test group) there was persistent statistically significant decrease in pain score until 1 month postoperatively then the decrease was statistically insignificant after 3 and 6 month postoperatively. In group 2 (placebo control group) there was statistically insignificant decrease after 1 week followed by insignificant increase after 2 and 3 weeks followed by insignificant decrease after 1 month followed by insignificant increase after 3 and 6 months Fig 7 .

\section{Mouth opening:}

Maximum mouth opening parameters were statistically non-significant between both groups preoperatively. The mouth opening scores in group 1 (laser study group) was statistically significantly higher than group 2 (placebo control group) after 1 week postoperatively until the end of the follow up period. In group 1there was persistent statistically significant increase in mouth opening until the end of follow up period after 6 months. This increase was statistically significant after 6 month postoperatively.

In group 2: There was statistically insignificant increase after 1 week followed by insignificant decrease after 2 weeks followed by very slight insignificant increase after 3 weeks followed by insignificant decrease after 1 month followed by insignificant increase after 3 and 6 months. The mouth opening did not improve significantly after 6 month postoperatively (Fig. 8) 
TABLE (1) One-way ANOVA comparing pain score in both group through the follow up period

\begin{tabular}{|c|c|c|c|c|c|c|}
\hline \multirow{2}{*}{ Pain Scores } & \multicolumn{2}{|c|}{ Group 1} & \multicolumn{2}{|c|}{ Group 2} & \multirow{2}{*}{ "t" } & \multirow{2}{*}{ Probability } \\
\hline & Mean & St Dev & Mean & St Dev & & \\
\hline Pre & 70.54 & 16.25 & 66.54 & 15.21 & 0.804 & $0.427 \mathrm{NS}$ \\
\hline 1 week & 52.13 & 16.29 & 65.28 & 16.35 & 2.548 & $0.015 *$ \\
\hline 2 week & 36.70 & 17.84 & 67.86 & 15.69 & 5.866 & $0.0001 *$ \\
\hline 3 weeks & 16.85 & 13.60 & 68.15 & 17.05 & 10.519 & $0.0001 *$ \\
\hline 1 month & 7.50 & 10.21 & 64.68 & 14.38 & 14.500 & $0.0001 *$ \\
\hline 3 months & 6.13 & 11.70 & 65.30 & 15.90 & 13.405 & $0.0001 *$ \\
\hline 6 months & 4.48 & 9.85 & 65.75 & 16.25 & 14.420 & $0.0001 *$ \\
\hline $\mathrm{F}$ & \multicolumn{2}{|c|}{68.823} & \multicolumn{2}{|c|}{0.144} & & \\
\hline Probability & \multicolumn{2}{|c|}{0.000} & \multicolumn{2}{|c|}{0.990} & & \\
\hline LSD & \multicolumn{2}{|c|}{7.346} & \multicolumn{2}{|c|}{8.322} & & \\
\hline
\end{tabular}

TABLE (2) One-way ANOVA comparing mouth opening in both group through the follow up period

\begin{tabular}{|c|c|c|c|c|c|c|}
\hline \multirow{2}{*}{$\begin{array}{c}\text { Mouth } \\
\text { Opening }\end{array}$} & \multicolumn{2}{|c|}{ Group 1} & \multicolumn{2}{|c|}{ Group 2} & \multirow{2}{*}{ " $t "$} & \multirow{2}{*}{ Probability } \\
\hline & Mean & St Dev & Mean & St Dev & & \\
\hline Pre & 36.30 & 7.90 & 37.15 & 7.04 & 0.359 & $0.721 \mathrm{NS}$ \\
\hline 1 week & 37.05 & 6.89 & 38.84 & 8.76 & 0.718 & $0.477 \mathrm{NS}$ \\
\hline 2 weeks & 40.25 & 6.80 & 36.63 & 6.84 & 1.679 & $0.102 \mathrm{NS}$ \\
\hline 3 weeks & 42.35 & 5.80 & 36.83 & 6.94 & 2.828 & $0.007 *$ \\
\hline 1 month & 43.55 & 4.73 & 36.65 & 7.12 & 3.610 & $0.001 *$ \\
\hline 3 months & 43.65 & 5.32 & 38.23 & 6.95 & 2.769 & $0.009 *$ \\
\hline 6 months & 44.35 & 4.54 & 39.15 & 6.15 & 3.042 & $0.004 *$ \\
\hline $\mathrm{F}$ & \multicolumn{2}{|c|}{5.79} & \multicolumn{2}{|c|}{0.545} & & \\
\hline Probability & \multicolumn{2}{|c|}{0.0000} & \multicolumn{2}{|c|}{0.841} & & \\
\hline LSD & \multicolumn{2}{|c|}{3.21} & \multicolumn{2}{|c|}{3.574} & & \\
\hline
\end{tabular}

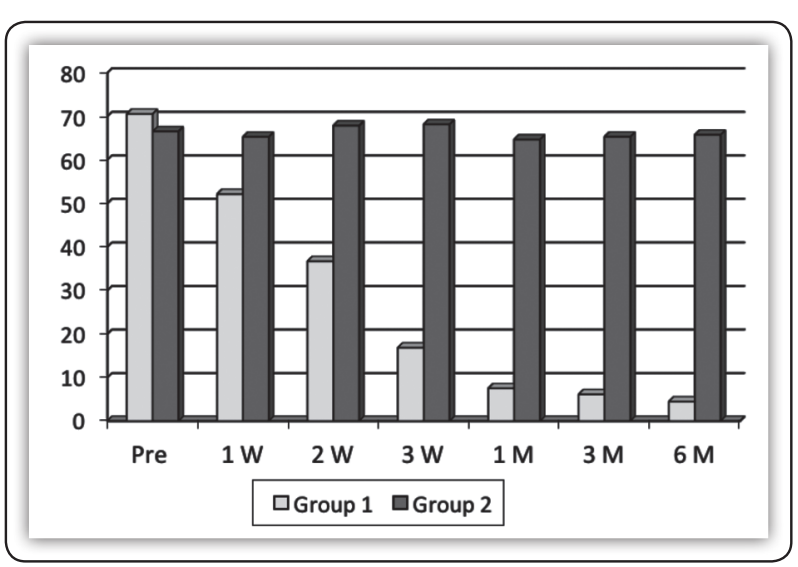

FIG (7) Mean values of pain scores in both group throughout the follow up period.

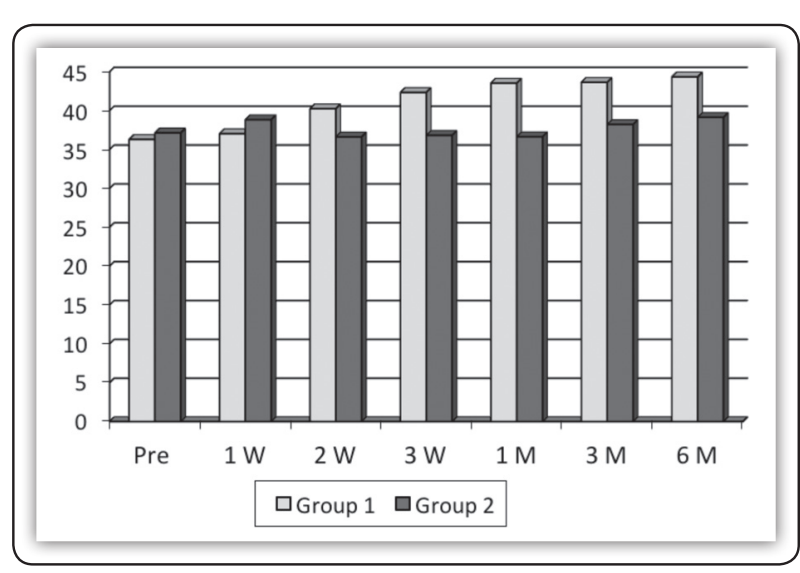

FIG (8) Mean values of mouth opening in both groups throughout the follow up period. 


\section{DISCUSSION}

In the TMD, the most common TMJ clinical findings are pain and limited mouth opening. The Effectiveness of Physiotherapy in the Management of TMD is well documented. Physical therapy including exercises, manual therapy, thermal therapy, electrophysical therapy like transcutaneous electric nerve stimulation (TENS) and acupuncture. Physiotherapy is intended to relieve musculoskeletal pain, reduce inflammation, and restore oral motor functions. ${ }^{(21)}$ Low level laser therapy represent additional physiotherapy options in the management of TMD. ${ }^{(22,23)}$

The analgesic effect of LLLT acts at different levels and by different mechanisms. Some explanations of this effect are: it increases betaendorphin level in spinal liquor, increases urinary excretion of glucocorticoids, which is a betaendorphin synthesis inhibitor, , decreases histamine and acetylcholine release, reduces bradykinin synthesis, increases adenosine tri phosphate (ATP) production, improves local microcirculation, increases lymphatic flow thus reducing edema. ${ }^{(24)}$.

LLLT has biologic effects, such as increased pain tolerance, due to changes in the potency of the cellular membrane; vasodilatation; reduction of edema; increase in intracellular metabolism; and acceleration of wound healing. The biostimulation effect of LLLT improves local microcirculation and oxygen supply to hypoxic cells in the painful areas. Simultaneously, tissue asphyxia is reduced to a minimum and collected waste products are removed. The laser-induced normalization of microcirculation interrupts the vicious cycle that originates, develops, and maintains pain; in addition, it restores the normal physiological condition of the tissue. Research has shown that LLLT can modulate inflammation by reducing the levels of biochemical markers (prostaglandin E2, messenger ribonucleic acid cyclooxygenase- 2 , interleukine- $1 \mathrm{~b}$, and tumor necrotizing factor-a), neutrophil influx, oxidative stress, edema, and hemorrhage in a dose-dependent manner. ${ }^{(25 \& 26)}$

Considerable works has been done to determining the effects of LLLT on pain and dysfunction of TMJ with varying results. Several studies have reported the positive effect of LLLT in TMD. ${ }^{27,28}$ Kulekcioglu et al. showed that, after 15 sessions of LLLT, the TMD of both myogenic and arthrogenic origins causes response to therapy with a significant reduction in pain, improvement in mouth opening and lateral movement, and diminished number of trigger points. ${ }^{(2)}$

Results differ from one study to the other, because there are a large range of treatment parameters in therapy (i.e., wavelength, power output, intensity, exposure time, total duration of treatment etc. ${ }^{(29)}$ In this study the protocol of laser application was carried out on six sessions twice weekly for 3 consecutive weeks with wavelength: $904 \mathrm{~nm}$, mean output power: $0.6 \mathrm{~W}$, duration: 60 seconds, dosage: $6 \mathrm{~J} / \mathrm{cm}^{2}$. The treatment period considered reasonable to keep the patients compliance and avoidance of patient's escapement if the period of treatment was prolonged. Parameters were recorded before and immediately after 1,2 and 3 weeks, 1, 3 and 6 months from the first session. In this study the results obtained showed that there were statistically significant improvement in pain and mouth opening in all cases of the laser group and remained so even in the follow-up period rather than the control group. The same protocol was used by Nabeel Sayed et al in twenty patients single study group. ${ }^{(20)}$ They concluded that LLLT promoted satisfactory results in reducing the pain intensity, number of tender points, joint sounds and improvement in the range of jaw motion. They also recommended further research using a large sample size and a control group to assess and compare the effectiveness of LLLT in TMD, which was accomplished in this study. 
LLLT is a non-invasive, reversible therapy without any known side effects. Thus, it is quite proper for TMD treatment. ${ }^{(27)}$ This augmented by the present study in which patients included were comfortable with the procedure and satisfied by the end results and had a better life style following the LLLT.

\section{CONCLUSION}

Within the limits of this placebo-controlled study, LLLT is an effective treatment for reducing pain, and improving limited mouth opening associated with TMD. The non-invasive and easy application of this therapy, supported its use as an alternative important option to other conventional treatment modalities in TMD. Further studies should investigate improved parameters in larger series.

\section{REFERENCES}

1. De Leeuw R. Orofacial Pain: Guidelines for Assessment, Diagnosis, and Management. 4th ed. Chicago: Quintessence; 2008.

2. Kulekcioglu S, Sivrioglu K, Ozcan O, Parlak M. Effectiveness of low-level laser therapy in temporomandibular disorder. Scand J Rheumatol 2003; 32:114-8.

3. Dimitroulis G. Temporomandibular disorders: a clinical update. Br Med J 1998; 317:190 -6.

4. Oshiro T, Calderhead RG. Low level laser therapy: a practical introduction Chichester: Wiley \& Sons; 1998.

5. Cetiner S, Kahraman S, Yu SU. Evaluation of lowlevel laser therapy in the treatment of temporomandibular disorders. Photomed Laser Surg 2006 ; 24: 5-10.

6. Fika H, Dosta T, Navra L, Klaschka J. Effectiveness of low-level laser therapy in temporomandibular joint disorders: a placebo-controlled study. Photomed Laser Surg 2007; 25:297-303.

7. Mazzetto M, Carrasco T, Bidinelo E, Andrade R, Mazzetto R. Low intensity laser application in temporomandibular disorders: a phase I double-blind study. Cranio 2007; 25:186-200.

8. Frare J, Nicolau R Clinical analysis of the effect of laser photobiomodulation (GaAs-904 nm) on temporomandibular joint dysfunction. Rev Bras Fisioter 2008; 12:37-41.
9. Carrasco T, Mazzetto M, Mazzetto R, Mestriner W. Low intensity laser therapy in temporomandibular disorder: a phase II double-blind study. Cranio 2008; 26:274-79.

10. Lassemi E, Jafari S, Motamedi M, Navi F, Lasemi R. Lowlevel laser therapy in the management of temporomandibular joint disorder. J Oral Laser Appl 2008; 8:83-87.

11. Santos T, Piva M, Ribeiro M, Antunes A, Melo A, Silva E. Laser therapy efficacy in temporomandibular disorders: control study. Braz J Otorhinolaryngol 2010;76:294-99.

12. Yu-Feng H, Hui-Wen Y, Yu-Hsien L, Chuan-Hang Y. Clinical effectiveness of laser acupuncture in the treatment of temporomandibular joint disorder Journal of the Formosan Medical Association 2014;113: 535-539.

13. Venancio D, Camparis C, Lizarelli F. Low intensity laser therapy in the treatment of temporomandibular disorders: a double-blind study. J Oral Rehabil 2005; 32:800-05

14. Emshoff R, Bo R, Pu E, Scho H, Strobl H. Low-level laser therapy for treatment of temporomandibular joint pain: a double-blind and placebo-controlled trial. Oral Surg Oral Med Oral Pathol Oral Radiol Endod 2008; 105:452-56.

15. Mayela B, Irene A, Janet C, Hugo A. Effectiveness of lowlevel laser as a treatment for pain management in arthrogenic temporomandibular disorders. Int Dent J Stud Res 2013;1:3-1-10.

16. Dworkin S, LeRresche L. Research diagnostic criteria for temporomandibular disorders: review, criteria, examinations and specifications, critique. J Craniomandib Disord. 1992; 6:301-55

17. Dym H, Israel H. Diagnosis and Treatment of Temporomandibular Disorders. Dent Clin N Am2012; 56: 149-61.

18. Kelly B. Nicole F., Lopes P., Robinsom V, Rogério F. low level laser therapy for temporomandibular disorders (TMD) treatment: A systematic review of randomized trials. JRD 2014; 2:376 -87.

19. Rahimi A, Rabiei S, Mojahedi S, and Kosarieh E. Application of Low Level Laser in Temporomandibular Disorders. Journal of Lasers in Medical Sciences 2011; 2: $165-170$.

20. Sayed N, Murugavel C, Gnanam A. Management of Temporomandibular Disorders with Low Level Laser Therapy. J. Maxillofac. Oral Surg. 2014; 13:444-450

21. Paço M, Peleteiro B, Duarte J, Pinho T. The Effectiveness of Physiotherapy in the Management of Temporomandibular Disorders: A Systematic Review and Metaanalysis. J Oral Facial Pain Headache. Summer 2016; 30(3):210-20 
22. Machado B, Mazzetto M, Da Silva M, de Felício C. Effects of oral motor exercises and laser therapy on chronic temporomandibular disorders: a randomized study with follow-up. Lasers Med Sci. 2016; 5:945-54.

23. Shukla D, Muthusekhar M. Efficacy of low-level laser therapy in temporomandibular disorders: A systematic review. Nat J Maxillofac Surg. 2016; 71:62-66.

24. Chang W, Lee C, Lin H, Hsu Y, Wang C, Lai P. A Metaanalysis of Clinical Effects of Low-level Laser Therapy on Temporomandibular Joint Pain. J Phys Ther Sci. 2014; 268:1297-300

25. Sandoval M, Mattiello-Rosa S, Soares E, Parizotto N. Effects of laser on the synovial fluid in the inflammatory process of the knee joint of the rabbit. Photomed Laser Surg 2009; 27: 63-9.
26. Bjordal J, Johnson M, Iversen V, Aimbire F, Lopes-Martins R. Photoradiation in acute pain: a systematic review of possible mechanisms of action and clinical effects in randomized placebo-controlled trials. Photomed Laser Surg 2006;24: 158-68.

27. Nunez S, Garcez A, Suzuki S, Ribeiro M. Management of mouth opening in patients with TMD through low level laser therapy and transcutaneous electrical neural stimulation. Photomed Laser Surg 2006; 24:45-9.

28. Ilbuldu E, Cakmak A, Disci R, Aydin R. Comparation of laser, dry needling, and placebo laser treatment in myofascial pain syndrome. Photomed Laser Surg 2004; 22:306-11.

29. Bjordal JM, Couppe C, Chow RT. A systematic review of low level laser therapy with location-specific doses for pain from chronic joint disorders. Aust J Physiother 2003;49:107-16. 